\title{
Entrelacs
}

Cinéma et audiovisuel

$8 \mid 2011$

Imaginaire

\section{Le bouleversement du territoire du film de genre. La Vengeance des femmes de Quentin Tarantino}

\section{Claudine Le Pallec Marand}

\section{(2) OpenEdition}

\section{Journals}

Édition électronique

URL : http://journals.openedition.org/entrelacs/228

DOI : 10.4000/entrelacs.228

ISSN : 2261-5482

Éditeur

Éditions Téraèdre

\section{Édition imprimée}

Date de publication : 1 février 2011

ISBN : 978-2-360850-03-7

ISSN : 1266-7188

\section{Référence électronique}

Claudine Le Pallec Marand, « Le bouleversement du territoire du film de genre. La Vengeance des femmes de Quentin Tarantino », Entrelacs [En ligne], 8 | 2011, mis en ligne le 01 août 2012, consulté le 20 avril 2019. URL : http://journals.openedition.org/entrelacs/228 ; DOI : 10.4000/entrelacs.228

Ce document a été généré automatiquement le 20 avril 2019

Tous droits réservés 


\title{
Le bouleversement du territoire $\mathrm{du}$ film de genre. La Vengeance des femmes de Quentin Tarantino
}

\author{
Claudine Le Pallec Marand
}

«Il n'y a guère, au fond, que deux grandes façons

de faire la théorie et l'histoire du cinéma :

l'ontologique et l'anthropologique. La première a pu d'abord sembler un peu courte, avec l'obsession

de la pensée indicielle- l'image de film comme trace de la réalité, et rien d'autre ; (...) Mais cela de toute façon ne suffit pas à faire droit à l'action du cinéma sur nos imaginaires, à faire droit à cette part proprement mythique qui a toujours été sienne et qui en a largement déterminé l'histoire.

Cet écart entre l'indicatif et l'optatif, entre les « choses qui sont là » et qu'il est vain de vouloir manipuler (Rossellini), et le monde « qui s'accorde à nos désirs » (Mo urlet), c'est l'écart producteur de mythes, la distance entre les deux composantes du mythique : le désir de comprendre le monde (tel qu'il est, hors de nous, malgré nous, sans nous) et

le désir de vivre bien dans le monde (d'y être réconforté, rassuré s'il le faut, d'y être guidé). ${ }^{1}$ »

Jacques Aumont

Question de méthode : L'imaginaire du féminisme est-il soluble dans la mise en scène?

Cinéaste-cinéphile, scénariste dialoguiste et réalisateur reconnu, Quentin Tarantino dirige ses équipes à l'intérieur même du système de studio qui fut le cadre de définition proposé par Laura Mulvey: le film hollywoodien comme instrument de domination patriarcale (cf. l'article historique de référence de la théorie féministe du cinéma de 1975 
«Visual Pleasure and Narrative Cinema $\left.»^{2}\right)$. Kill Bill 1, Kill Bill 2 et Boulevard de la mort, à l'audience populaire indéniable, ont la particularité de développer le récit du bon droit moral et de la vengeance sanguinaire d'une ou de plusieurs héroïnes que l'art et la manière $\mathrm{du}$ réalisateur désigne comme l'adjudante cathartique du spectateur. On peut aussi considérer Jackie Brown, le film qui les a précédés, comme l'histoire d'une femme noire américaine décidée à prendre sa revanche contre le sort qui la destinait à être manipulée de tous côtés par des hommes (son commanditaire mafieux et le chantage de deux policiers qui la surveillent). Ces quatre films croisent aisément le procès de principe ${ }^{3}$ qui se réfère principalement aux arguments du mélange des genres cinématographiques, aux conventions du regard sexué au cinéma, mais aussi sur le trompe l'œeil que constitue l'inversion terme à terme du pouvoir et des caractérisations (principalement le rapport à la violence) des personnages, selon qu'il soit homme ou qu'il soit femme (et qui résume superficiellement les films cités).

3 Disponible en français grâce à la revue Trafic (numéro 50), il existe pourtant un texte tardif de Laura Mulvey daté de 2004, peu commenté, invitant à reconsidérer le cinéma populaire. Cet article répondait à une commande autour de la question "Qu'est-ce que le cinéma? ». Il est consacré aux " Quatre premiers plans d'Imitation of Life » de Douglas Sirk. Le scénario du film raconte la solidarité de deux mères célibataires, Lora, l'actrice blanche, qui emploie à son service la seconde, Annie, une jeune femme noire. Laura Mulvey précise sa méthode: "Si on arrête la scène, qu'on revient en arrière, qu'on la répète, l'iconographie et la topographie sont facilement identifiables et la structure d'une scène intégralement faite de symétrie et d'opposition acquiert une signification esthétique et cinématographique propre ${ }^{4} »$. La liaison inédite entre la revendication d'une thématique (rapports de classe, de race et de sexe- exemplifiés dans l'opposition et la solidarité des deux héroïnes d'Imitation of Life) et un savoir faire attentif au cinéma (la description des choix du cinéaste par Laura Mulvey) recoupe la démocratisation de la vision fragmentée et répétitive favorisée par le DVD.

Posons l'existence d'un continuum problématique du cinéma et du féminisme, qui croise toutes sortes de définitions et de pratiques du cinéma et qui s'échelonne entre deux pôles. Le premier est thématique, c'est la représentation autodéclarée (propos du cinéaste) ou nominative (y compris la ridiculisation du mouvement ${ }^{5}$ qui perpétue un thème ancien); l'autre est esthétique, ce sont des formes cinématographiques, soit de figuration soit d'intégration des énoncés et visibles féministes, chers à ceux qui pensent qu'il est possible de mettre en scène la différence des sexes avec des images et des sons. Les instruments du second pôle, le pôle esthétique, sont les formes même du cinéma (genres cinématographiques, mouvements de caméra, image ET son...).

5 Il s'agit ainsi de rendre compte de la manière dont Quentin Tarantino est capable de proprement réinventer les genres cinématographiques autour de la violence, dans l'opération du filmage, dans la façon dont il modifie le cadrage, la composition de l'image, la mise en sc_e8ne et les agencements audiovisuels. En marge des apports psychanalytiques mais sans pour autant être quitte de l'attention nécessaire aux caractéristiques narratives (la vengeance d'une femme et l'instauration de son émancipation) et spectatorielles (le plaisir de la vision de la violence au cinéma, la catharsis), le cinéma de Quentin Tarantino pourrait être celui d'une mise en scène politiquement bouleversée du territoire cinématographique du film de genre. 


\section{Le socle de l'imaginaire du cinéma de Tarantino : le cercle parfait d'une communauté}

6 Pour se convaincre de l'intérêt du cinéma de Quentin Tarantino quant à l'imaginaire du film de genre, il est troublant de découvrir une même manière de filmer les dialogues dans son avant-dernier et dans son premier film, Reservoir Dogs où pas une seule femme n'est à l'écran, (une serveuse exceptée). La teneur des dialogues y interroge explicitement le fait de «faire communauté » et notamment, une communauté d'hommes ou de femmes.

7 Le champ contre-champ pour filmer les dialogues est un système réputé pour soutenir la confrontation des personnages en effaçant arbitrairement leur proximité. Immanquablement, le montage des dialogues de Reservoir Dogs mime l'idée que la parole peut-être une âpre confrontation. Dans le pré-générique où tous les personnages sont réunis autour d'une table de fast food, l'un d'entre eux propose de « tuer » (mime à l'appui) son contradicteur. Ce personnage, ostracisé pour avoir refusé de laisser un pourboire, s'insurge plus tard dans le seul flash back "collectif" (et non plus communautaire) où seront réunis à nouveau tous les hommes du film, avant l'échec lamentable du braquage. A ce moment là, il refuse que le chef de l'opération attribue arbitrairement à chaque braqueur un patronyme: Mister Brown, Mister White, Mister Blonde, Mister Blue, Mister Orange et Mister Pink. La question des identités, censée être contournée pour protéger l'anonymat des truands, revient instantanément en guise d'étiquette sociale (le nom comme signe potentiel d'un sexe, d'une sexualité et d'une race). Le chef clôt ce dialogue, à première vue pas très sérieux, en précisant que tout le monde veut être «Mister Black» et que ce genre de réunion n'est pas une réunion politique (« city council meeting »).

Si la critique a depuis longtemps mesuré la crudité et la boulimie de paroles du cinéaste, elle refoule jusqu'ici la perspective politique de sa cinéphilie. Branché sur les ondes des années 70 (la radio de Reservoir Dogs), Quentin Tarantino n'a jamais cessé de creuser la problématique identitaire du cinéma grindhouse $e^{6}$ des années 70 .

9 La séquence pré-générique qui inaugure Reservoir Dogs réunit les huit personnages masculins du film tandis que la suite les sépare, excepté le court flash back qui exacerbe la dissension du groupe. Avant que les champs contre-champs redoublent les confrontations « identitaires » des dialogues, le prologue de Reservoir Dogs amorce dans un premier temps plusieurs petits panoramiques qui anticipent la prouesse technique de son dernier film: "Je pense toutefois qu'un des meilleurs plans que j'ai jamais tournés - un des plus complexes et des mieux exécutés - est le plan sans coupe à $360^{\circ}$, autour de la table, dans Boulevard de la mort. Je ne suis jamais allé aussi loin dans le tour de force. $»^{7}$ : prouesse technique sans aucun doute, et pas n'importe quand dans le film. Cette mise en scène sans heurts redouble une qualité d'écoute et de cohésion particulière qui compose visuellement la communauté à un moment précis de chacun des deux films. Le prototype de ce panoramique circulaire pour filmer les dialogues crus et amicaux d'une communauté exclusive (d'hommes dans Reservoir dogs, puis de femmes dans Boulevard de la mort), se trouve dans Reservoir Dogs. Cette figure du lien est ainsi utilisée au moment où la conversation n'est pas belliqueuse, lorsque la tension n'est pas encore (ou jamais) présente. 
10 L'aube du prologue de Reservoir dogs écarte d'abord les occasions de dissension des sujets de conversation sur le gouvernement, les syndicats et les conditions de travail des serveuses. Quentin Tarantino intervient le premier avant même l'image. Sa voix (à l'intonation aigüe singulière, à l'époque encore inconnue) affirme de manière péremptoire : "Let me tell you what Like a Virgin is about. » Son personnage explique que cette célèbre chanson de Madonna est l'histoire d'une « machine à baiser » qui retrouve la douleur qu'elle n'avait plus jamais ressentie depuis qu'elle n'était plus vierge, et résume la chanson à « une métaphore sur les grosses queues ». Un autre préfère y voir l'histoire d'« une fille très vulnérable » qui « a baisé à droite et à gauche et qui rencontre un garçon sensible ». La chanson pop de Madonna dans le cinéma de Quentin Tarantino fonctionne comme une mise en abyme d'un possible de la vocation de l'art populaire. La possibilité de réception, et donc d'interprétation multiple des représentations, fondement problématique des cultural studies, sous-tend le cinéma de Quentin Tarantino. La médiation culturelle permet à chaque individu de mimer et résorber les conflits qui ne se transforment jamais en conflits réels, à l'inverse de la tension palpable des sujets de conversation politique filmés en champ contre-champ. Le jugement sur la chanson de Madonna diverge selon les personnages, pour autant les deux interprétations proposées ne s'excluent pas mutuellement. Alors que l'un rend équivalent des mots crûs et des actes, et résume la chanson par des détails d'ordre sexuels (« sex machine», «virgin»), l'autre préfère énoncer un jugement moral à travers une caractérisation psychologique du personnage de la chanson ("sensible girl», «nice fellow »). Cette opposition rhétorique est une sorte de scène inaugurale pour qui se risque à une étude de l'imaginaire des films de Quentin Tarantino. Or, le flot de langage ordurier et intimiste est un outil à la disposition d'une incarnation cinématographique inédite de la maîtrise féminine des mots, de la valorisation sexuelle et de la présentation sociale de son propre corps.

11 Boulevard de la mort est construit en forme de diptyque, l'un au Texas, l'autre au Tennessee, à partir du parcours similaire de deux quatuors féminins, cibles du désir sexuel du cascadeur Stuntman Mike, par automobile interposée. Pour prolonger notre étude sur l'articulation entre filmer les dialogues et filmer l'espace et les liens d'une communauté issue de la mise en scène du prologue masculin de Reservoir Dogs, on peut scruter l'opposition des deux séquences de femmes attablées dans Boulevard de la mort. Leur distinction formelle accompagne et donne sens au diptyque.

12 "Chez Guerros », premier bar du premier trio, la discussion est filmée sous la forme d'un champ contre-champ classique qui va révéler une fausse note dans le groupe. Bien que par la suite l'humour du dialogue dissipe le malaise soulevé, le collectif de la première partie de Boulevard de la mort n'est pas né sur un pied d'égalité. Dans le bar suivant, où elles ont rendez-vous avec des garçons, la mise en scène multiplie les scénettes individuelles. Jungle Julia s'isole pour envoyer des textos à son petit ami et Butterfly prend l'air avant que ses deux amies se désintéressent de son show devant Stuntman Mike. Pour finir, faute peut-être de cohésion dans le groupe, les trois femmes n'atteindront jamais leur destination finale.

13 Le fameux plan de $360^{\circ}$, dont on a repéré le prototype et isolé la fonction, est réservé au repas des quatre héroïnes suivantes de Boulevard de la mort, dont les thèmes de conversation recoupent une fois de plus culture puis politique. Parlant de culture populaire, elles s'interrogent sur l'opposition traditionnelle des publics de film de genre en comparant un film de course poursuite (Vanishing point, Richard C. Sarafian, 1971) et un teenmovie (Pretty in Pink, Howard Deutch, 1986). Le panoramique circulaire de la 
communauté exclusivement féminine résiste cette fois-ci quand la discussion passe au sujet du port d'arme aux Etats-Unis. Le sujet divise pourtant la tenante de l'argument humaniste, pour qui la possession d'une arme accroît les possibilités d'homicides, et la tenante de l'argument différentialiste qui met en avant la hiérarchie sexuée des statistiques d'agressions physiques. La cohésion amicale de la communauté féminine représentée est telle que les stéréotypes identitaires sont mêmes ici clairement exorcisée par l'humour: "Tu ne traites pas un kiwi de Kangourou!», résume la distinction entre l'Australie et la Nouvelle Zélande.

14 Ce mémorable $360^{\circ}$ filme non seulement la communauté féminine du film mais, si l'on est très attentif, la présence du personnage de Kurt Russel (Stuntman Mike), de dos, bord cadre, en arrière-plan, assis à une chaise de comptoir. En quoi cette présence périphérique (mais non invisible) du personnage masculin traduit contre quoi doit se défendre la communauté féminine soudée du cinéma de genre de Quentin Tarantino?

\section{Purger le film de genre de son sexisme (Jackie Brown, Boulevard de la mort)}

Le cinéaste se confronte au problème du sexisme inhérent aux films d'action dans les deux films qui encadrent le binôme kill billien. En effet, la place tutélaire des personnages masculins principaux est largement remise en cause dans les séquences qui introduisent Ordell (Jackie Brown) et Stuntman Mike (Boulevard de la mort). Criminel notoire, pour lequel le cinéaste n'est pas avare de bons mots, qu'il distribue toujours sans distinction de sexe, ni de morale, l'anti-héros masculin de Quentin Tarantino est en fait identifié au sexisme traditionnel du genre cinématographique que son personnage incarne.

16 Avant de voir le visage d'Ordell et d'entendre sa voix, la séquence qui le présente commence par un programme de télévision de vente d'armes. Ce programme a un nom ( « Chicks who loves guns ») et le spectateur comprend instantanément sa facture visuelle : une belle et jeune femme en bikini pour chaque séquence dédiée à une arme différente avec des cadres d'image qui privilégient les seins et les cuisses. La greffe misogyne des images de la séquence caractérise le personnage. Les dialogues des personnages féminins du film achèvent d'ancrer son profil tout en dénonçant les limites de son autorité terroriste: Mélanie (une jeune californienne blonde, dont il attend une soumission de rigueur) suggère à son ami Louis que la logorrhée d'expert armurier d'Ordell est un plagiat éhonté ; Simone, une autre de ses "protégées", disparaît littéralement du film et obtient a insi son indépendance après avoir dérobé son argent; et Jackie donne un peu d'argent à Mélanie en lui affirmant qu'Ordell n'a jamais rien fait pour les femmes dont il s'entoure, uniquement pour son business et son plaisir.

17 La longueur adéquate du plan qui permet à la voiture de Stuntman Mike de prendre la suite, derrière celle des filles, reconduit la trame de la menace que fait courir le personnage masculin épiant les personnages féminins. Dans la seconde partie qui se déroule au Tennessee, les indices de cette menace, à ce moment bien connu du spectateur, précèdent la présentation du second collectif féminin. L'anti-héros avance désormais à découvert, sans plus aucune précaution, fort du soutien du désir du spectateur puisqu'il détermine la répétition de la menace (et avec elle le prolongement de l'histoire et du plaisir). La reconduction du pervers misogyne dans la fiction est auréolée de l'éclat gore de la mise en scène précédente où le cinéaste réitère l'impact meurtrier sur 
chacun des quatre corps féminins déchiquetés par le choc. L'impunité juridique du personnage sur laquelle ironisent le duo de marshals, est une convention scénaristique qui permet à Quentin Tarantino la mise en scène flamboyante du retour du personnage. Radio à plein volume, pneus grinçants, lunettes noires, arrivée en plein jour et changement de focale selon la direction du visage de Stuntman Mike, attiré par la voiture des filles qui se gare juste à côté de lui, pointent la licence mortifère dont bénéficie le personnage masculin dans ce type de films. Le sens de la seconde partie de Boulevard de la mort naît justement du bouleversement du plaisir du spectateur au profit de «la vengeance des femmes ». Abernathy et Lee sont loin de s'inquiéter. Le contre-champ qui prend en charge le regard des jeunes femmes dessine le ridicule de l'auto-mise en scène de Mike avec un commentaire en forme de portrait robot dérisoire du serial killer : «little dick». La verbalisation anticipe la capacité des héroïnes à intervenir contre cet homme. En fait, la nature du crime est énoncée pour la seconde fois : «C'est sexuel. Impact à grande vitesse, métaux tordus, verres brisés. Sans doute la seule manière pour ce diabolique dégénéré de ce vider les couilles. » -avait déjà expliqué le marshal-. L'anti-héros est incontestablement un prédateur sexuel qui ne peut imaginer que des filles jouissent sans lui. C'est l'idée que détourne la séquence humoristique où les trois héroïnes audacieuses et sans peur de Boulevard de la mort abandonnent leur quatrième comparse costumée pour les besoins d'un "chear leader's movie». Elle est ainsi “joyeusement" sacrifiée au désir commun d'aventures des trois autres pour être livrée à la concupiscence d'un fermier redneck, et tout aussitôt sortie du film.

18 Il est indéniable que dans chacun de ces quatre films de la « vengeance des femmes » (à suivre), il existe un commanditaire masculin. Ici trône la présence masculine envoûtante de Bill, encore fragmentaire (une voix profonde, des mains fortes) dans Kill Bill 1. L'image du maître masculin se subdivise dans les «chapitres » d'apprentissage d'arts martiaux de Kill Bill 2 avec Pai Mei et Hattori Hanzo. Leurs enseignements de kung fu et de sabre vont permettre à l'héroïne Béatrix Kiddo de parvenir à tuer Bill, son ancien maître à penser dans le monde du crime. L'ambiguïté de cette figure s'explique par le désir narratif du spectateur qui est tout autant focalisé sur Bill (son apparition dans la mise en scène) qu'il est littéralement comblé par le talent de conteur du personnage. Ainsi, la mise en scène peut s'attendrir sur ce personnage sanguinaire de cow boy mourant au moment de sa mise à mort finale, en musique, et même pieds nus (détail d'ordinaire réservé aux femmes dans le cinéma de Tarantino). Bill est logiquement une figure positive puisqu'il est à l'origine même du désir de film d'action : il a raconté des histoires à Béatrix et à sa fille, à qui il donne symboliquement un pistolet en plastique et lui laisse regarder (avec l'accord de sa mère) Shogun assassin (Robert Houston et Kenji Misumi, 1980) avant de s'endormir. Bill est le grand méchant de Kill Bill mais ce n'est pas un personnage misogyne comme Ordell ou Stuntman Mike.

\section{Maîtrise versus spectacularisation des corps féminins (Kill Bill Vol 1 et 2, Jackie Brown et Boulevard de la mort)}

Le succès final de l'hôtesse de l'air Jackie Brown est clairement associé à la logique de supériorité intellectuelle du personnage, visible lors de sa première confrontation avec Ordell, qui l'emploie pour rapatrier son argent caché au Mexique. Alors que la technique du split screen peut suggérer au spectateur que le second personnage masculin pourrait la protéger, la mise en scène déçoit cette logique au profit d'une autre, plus subtile. Le 
regard surpris de Max qui découvre que sa boite à gants est vide fait écho à un bruit de déclic où Jackie utilise l'arme qu'elle lui a dérobée pour la "braquer sur la queue » d'Ordell qui préméditait de la tuer. La source du renversement sexuel au sein des conflits est clairement identifiée à une captation féminine de l'initiative sexuelle et de l'activité (la femme est moteur de l'action). En témoigne également la longue séquence emblématique à l'hôpital de la renaissance de l'héroöne de Kill Bill 1. Quentin Tarantino insiste sur l'acharnement dont elle fait preuve pour recouvrer ses sens et sa volonté et mettre un terme aux viols successifs grâce à l'assassinat de son tortionnaire. Aux fins de poursuivre sa vengeance à l'encontre des responsables de son coma, un clin d'œil particulièrement cynique l'attend. En effet, pour quitter rapidement l'hôpital, elle doit utiliser la voiture du défunt sadique, affublée du nom de « Pussy Mobile » par son ancien propriétaire. S'agit-il seulement d'inverser la balance de la hiérarchie patriarcale (sans aucune sortie possible?) pour établir une seconde sor te d'équilibre continu de domination et de pouvoir chez un réalisateur qui a dévolu son cinéma à la représentation/interprétation/valorisation de la violence inhérente au cinéma de genre.

Afin de contourner la limite de toute lecture de «l'inversion» au cinéma, il est plus pertinent d'étudier le cinéma de la "vengeance des femmes » depuis la question de la mise en scène plutôt que de leur indéniable supériorité scénaristique. Nous allons l'aborder d'abord sous l'angle de la maîtrise du corps et enfin sous l'angle de sa spectacularisation (introduction d'un show, d'un spectacle dans le film).

Les scénarios de Kill Bill I et II sont entièrement construits autour de la maîtrise du corps que partage l'héroïne avec ses ennemies de la Deadly Viper Assassination Squad. La maitrise du corps et de la force n'ont rien d'une transgression ou d'une monstruosité dans l'univers de Kill Bill. Au-delà même de la multiplication des personnages féminins agiles et violents, plusieurs séquences de Kill Bill mettent littéralement en scène le corps féminin en prise avec le cadre de l'écran de cinéma. Dans des plans de Kill Bill 2, dont le haut du cadre est borné par le couvercle du cercueil, le spectateur est enfermé avec l'héroïne sous la terre, avant sa « résurrection » terrestre. On peut aussi citer dans Kill Bill 1, la scène du restaurant où l'héroïne, venue se venger d'Oren-Ishii, sera confrontée aux Crazy 88 qui composent sa garde rapprochée. Tels des personnages de comics, les personnages féminins ont les sens humains et des capacités physiques surnaturelles. Le zoom avant sur le visage et le jeu de l'actrice (sursaut du regard) de la chef Yakusa soulignent les sens développés du cruel personnage en manifestant une ouïe quasi-fantastique. Le panoramique sur la position inconfortable de Béatrix au plafond puis, au plan suivant, ses pieds qui glissent depuis le haut du cadre, construisent l'image de personnages féminins complètement maîtres des disciplines et des nécessitées liées à de telles prouesses physiques.

En dehors de cette capacité physique qui permet de combler l'écart entre les personnages masculins et féminins et faire évoluer le statut traditionnel des femmes dans le film d'action, il est intéressant de se pencher sur les restes d'une scène féconde dans la théorie féministe du cinéma, la scène de strip-tease, comme point de référence de la spectacularisation du corps féminin au cinéma. Quentin Tarantino s'y confronte directement dans la scène de strip-tease de Simone devant Louis (Jackie Brown) et la scène de lap-dance dans la première partie de Boulevard de la mort.

23 Au-delà de l'âge certain des protagonistes de la première séquence repérée, c'est davantage encore la mise en scène, à travers un objet, un fauteuil à bascule, qui va permettre au cinéaste d'ironiser sur ce type de scène. Le strip-tease est filmé 
alternativement à partir de deux plans : tout d'abord, il cadre les fesses de la danseuse alors qu'à l'arrière-plan le personnage joué par Robert De Niro est avachi sur le fauteuil ; ensuite, le second est une sorte de contre-champ. En bas du cadre, les allers et retours de sa nuque finissent par donner une touche comique et touchante mais totalement désérotisée à la séquence qui s'achève avec un coup de fil et la sortie de la danseuse vers les toilettes.

Dans Boulevard de la mort, Quentin Tarantino ne reprend pas le choix radical qui consiste à désérotiser la séquence. Plusieurs plans isolent le postérieur de Butterfly et le cinéaste joue avec le cadre cinématographique au ras de ses fesses. Ce qui singularise définitivement cette scène, c'est qu'autour du siège de Stuntman Mike qui trône au milieu $\mathrm{du}$ bar, les spectatrices du lap-dance sont toutes des femmes. Cette scène s'achève abruptement comme un saut de photogramme d'un film à petit budget et coupe net l'érotisme (« déjà vu ») de la scène.

\section{Coup de force culturel ${ }^{8}$ féministe du cinéma de Quentin Tarantino}

La célèbre marque de fabrique de ses plans de pied nus féminins, et masculin avec Bill, est moins un nouvel avatar anatomique fétichiste que le trope exemplaire d'une autre manière de filmer. Dans le cinéma de Quentin Tarantino il ne faut pas refuser d'entendre que les personnages féminins insultent les hommes comme elles s'insultent entre elles, avec le même vocabulaire, formulent la cartographie de leur désir sexuel, doivent prendre la décision de mener à terme une grossesse, séduisent quand et qui bon leur semble, ne veulent pas dépendre des hommes, peuvent être excitées par le récit minutieux des frasques hétérosexuelles de leurs amies ou s'interrogent sur les conséquences symboliques de la position hétérosexuelle dominante : "qui était au-dessus? "...

L'un des coups de force culturel du cinéma de Quentin Tarantino est d'avoir intégré des éléments féministes dans la culture populaire cinématographique. Si le cinéaste réussit là où d'autres échouent et d'autres n'ont pas conscience de continuer à échouer, c'est que le principe d'inversion des pôles féminins et masculins n'est pas le guide ambigüe de ses scénarios, ni de sa mise en scène : l'incorporation du discours féministe est un enjeu majeur de l'originalité et de la maîtrise de son cinéma. Le credo du féminisme de Quentin Tarantino, c'est d'abord la mise en scène de la solidarité. Ensuite, les dialogues sur la sexualité sont prétextes à discussions inépuisables, aux bons mots et à l'affirmation de la maitrise de son corps. Enfin, la différence et la hiérarchie des sexes est un ressort des genres cinématographiques que le cinéma se doit de réinterroger inlassablement. Le cinéma de Quentin Tarantino est l'occasion d'affirmer que le féminisme n'est pas seulement un sujet thématique mais un enjeu de mise en scène: filmer les dialogues, filmer la hiérarchie des sexes, filmer l'amitié, les communautés, filmer la maitrise du corps, filmer la séduction des corps... 


\section{NOTES}

1. AUMONT Jacques, La différence des sexes est-elle visible? Les hommes et les fermmes au cinéma. Conférences du Collège de l'histoire de l'art cinématographique. 1999-2000, p7.

2. Cette étude sémiologique qui utilise les concepts psychanalytiques de fétichisme et de voyeurisme détermine des exemples de convergence des regards voyeuristes de la caméra, des champs contre-champs des regards des personnages masculins qui transforment les personnages féminins en objet et des regards du spectateur forcés de s'identifier aux deux précédents.

3. Par ailleurs introducteur dans les universités françaises et surtout artisan et pédagogue des présupposés épistémologiques des gender et cultural studies, avec la fidèle complicité professionnelle de Geneviève Sellier depuis leur ouvrage commun qui fait date La drôle de guerre des sexes du cinéma français (1930-1956), Noël Burch récuse d'avance toute étude, qui plus est au sein du cinéma populaire hollywoodien, qui viendrait nier (et non plus illustrer) l'imaginaire patriarcal. Le cinéma postérieur aux années 70 continue de nourrir l'anathème de l'auteur à travers la méfiance soutenue à l'égard du ton de l'ironie et la suspicion ouverte à l'égard du métissage des genres cinématographiques: "s'agissant d'un film d'auteur (y compris surtout des nouveaux auteurs hollywoodiens) l'ambigu, l'indécidable, l'hermétiquement dérisoire sont plus que jamais à la mode. (...) Cette habile fusion des genres, loin d'être un "simple " effet de style, accomplit donc une sorte de grand écart idéologique en même temps qu'elle constitue une stratégie de box-office auprès des deux publics mutuellement exclusifs. " (c'est nous qui soulignons) [BURCH Noël, De la beauté des latrines : pour réhabiliter le sens au cinéma et ailleurs, Paris, L'Harmattan, Collection Champs visuels, 2007, p166-167.]

4. MULVEY Laura, «Les quatre premiers plans d'Imitation of Life », Trafic, $n^{\circ} 50$, « Qu'est-ce que le cinéma?», p479.

5. Par exemple: ROLLET Brigitte, «Un canular anti-MLF : Calmos de Bertrand Blier », Un siècle d'antiféminisme, sous la direction de Christine Bard, Fayard, 1999, pp 379-390.

6. Grindhouse est le nom du film américain que Quentin Tarantino a réalisé avec Roberto Rodriguez comme un double programme sur le modèle du cinéma d'exploitation. Boulevard de la mort (Deathproof) est la version longue du premier programme réalisé par Quentin Tarantino. Le terme désigne des petits cinémas de quartier (souvent urbains) ou les drive-in qui, dans les années 60-70 diffusent des productions qui ne suivent pas les contraintes et les régulations édictés par les studios en ce qui concerne la représentation explicite du sexe et de la violence. Appelées «films d'exploitations » ou plus populairement "Grindhouse movies », ces films sont synonymes à la fois d'une forme de production (limitation des coûts, tournage très rapide, auteur•e interchangeable, détournements d'images de films...), de distribution (écrans et salles spécialisés) mais aussi de formules scénaristiques qui ont commercialement fait leur preuve : «blaxploitation", «sexploitation", «shock and horror» (drogue, violence et gore), Kungfuxploitation... selon les quatre genres discriminés dans le Hors série de Mad Movies de juin 2007 « Spécial Grindhouse. Sexe ! Horreur ! Action! Dans les veines du cinéma d'exploitation ». La dimension politique des propos des films d'exploitation est assurément liée à la prise en considération d'une certaine audience, et donc à des enjeux de prime abord commerciaux.

7. Quentin Tarantino, «Je ne sais rien faire de la main gauche » par Emmanuel Burdeau et Cyril Neyrat. Propos recueillis à Cannes le 24 mai 2007, Les Cahiers du cinéma, $\mathrm{n}^{\circ}$ 624, p 21.

8. Dans sa critique de Boulevard de la mort publié dans les Cahiers du cinéma, Emmanuel Burdeau sacrifie le sens de la narration et le sens des dialogues pour délimiter « le coup de force culturel » du film uniquement à l'aune du genre cinématographique : « Boulevard de la mort fait se succéder à 
grand train deux parties similaires[sic] qu'aucune raison ne raccorde, deux fois la même histoire d'un vieux loup » (...) « la course de Boulevard de la mort est d'abord celle de la pellicule. Les langues ont beau délirer » (...) «Il ne faut pas chercher ailleurs le coup de force culturel de Boulevard de la mort. Celui-ci tient certes à une duplication, mais ce n'est que secondairement celle de l'hommage rendu par un cinéaste de renom à un genre oublié. C'est bien davantage l'audace de faire se succéder des conversations, filmées de manière volontiers banales, et deux scènes de voiture- une collision, une poursuite- qui comptent déjà parmi les sommets du film d'action. " Le regard esthétique sensible au féminisme enrichit bien les études esthétiques. La spécificité de la mise en scène des différents personnages de part et d'autre de la rupture narrative du diptyque et l'omniprésence des dialogues sociosexués dans une mise en scène qui se préoccupe de «faire communauté » participent de la réussite de Boulevard de la mort dont le «coup de force culturel » est aussi d'avoir intégré des éléments de la vie quotidienne et de la vulgate de la théorie féministe dans la culture populaire cinématographique.

\section{RÉSUMÉS}

Repenser le mélange des genres cinématographiques chez Tarantino, et dépasser l'évidence de ce constat, à savoir, l'emprise des femmes dans son imaginaire, permet de rendre compte de la manière dont le réalisateur américain est capable de proprement réinventer la violence au cinéma : dans l'opération du filmage, notamment, dans la façon dont il modifie le cadrage, la composition de l'image, la mise en scène et les agencements audiovisuels. En marge des apports psychanalytiques dominés par le concept de la castration, mais sans pour autant être quitte de l'attention nécessaire aux caractéristiques narratives (la vengeance d'une femme et l'instauration de son émancipation) et spectatorielles (le plaisir de la vision de la violence au cinéma, la catharsis), le cinéma de Quentin Taran tino pourrait être celui d'une mise en scène politiquement bouleversée du territoire imaginaire du film de genre, menaçant les conventions du regard sexué au cinéma (soi-disant inexorablement reconduit par la logique des genres cinématographiques à destination d'un seul « sexe » de spectateur).

\section{AUTEUR}

\section{CLAUDINE LE PALLEC MARAND}

Doctorante, chargée de cours, Université de Paris VIII 